International Electronic Journal of Algebra

VOLume 20 (2016) 86-99

\title{
STRONGLY T-EXTENDING MODULES AND STRONGLY T-BAER MODULES
}

\author{
S. Ebrahimi Atani, S. Dolati Pish Hesari and M. Khoramdel \\ Received: 15 September 2015; Revised: 06 June 2016 \\ Communicated by Burcu Üngör
}

\begin{abstract}
In this paper, we introduce the notions of strongly t-extending and strongly t-Baer modules. We provide several characterizations and investigate properties of each of these concepts. It is shown that, while a direct summand of a strongly t-extending module inherits the property, direct sums of strongly t-extending modules do not. Moreover, when a direct sum of strongly t-extending modules is strongly t-extending, is investigated. Also, it is proved that every strongly t-extending module has strongly summand intersection property and densely co-Hopfian property.
\end{abstract}

Mathematics Subject Classification (2010): 16D70, 16D80

Keywords: Extending modules, strongly $t$-extending modules, Baer modules, strongly $t$-Baer modules

\section{Introduction}

The idea of investigating a mathematical structure via its representations in simpler structures is commonly used and often successful. The representation theory of extending modules and Baer modules has developed greatly in the recent years. It is an area which is very firmly based on the detailed understanding of examples, and there are many powerful techniques for investigating representations of particular extending modules (resp. Baer modules) and for relating representations of different extending modules (resp. Baer modules) to one another. One point of this paper is to introduce a subclass of t-extending modules (resp. t-Baer modules).

The notion of an extending module can be traced back to work of von Neumann in the 1930s. His interest in quantum mechanics led him to develop "continuous geometry", which we today refer to as upper and lower continuous complete modular lattices. In recent years theory of extending modules and rings has come to play an important role in the theory of rings and modules. A module $M$ is called extending if every submodule is essential in a direct summand. Many properties of extending modules have been introduced and studied by several authors. 
The Baer property for rings was first considered by Kaplansky [11]. A ring $R$ is called Baer if the right annihilator of any subset of $R$ is generated as a right ideal by an idempotent. The notion of Baer property in a general module theoretic setting has been introduced by Rizvi and Roman. In [13], a strong connection is established between extending modules and Baer modules.

In [3], Asgari and Haghany introduced the concept of t-extending modules and t-Baer modules by using second singular submodules. The notion of a strongly extending module is introduced in [9], which is a subclass of the class of extending modules. Motivated by definitions of strongly extending modules and t-extending modules, we introduce the notion of strongly t-extending modules which are particular t-extending modules and a generalization of strongly extending modules.

After some preliminaries in Section 2, we propose the definition of a strongly t-extending module in Section 3. We explore some equivalent conditions for a module to be strongly t-extending. It is shown that direct summands of strongly t-extending modules are too strongly t-extending. A natural question to ask, for strongly t-extending modules in whether the property is preserved by a direct sum of such modules. First we answer this in the negative by an example. Next we give a necessary condition for a sum of strongly t-extending modules to be strongly t-extending. Also it is shown that strongly t-extending modules are densely coHopfian.

We define and investigate strongly t-Baer modules in Section 4, which were motivated by definitions of t-Baer modules and Abelian Baer modules. We give characterizations of a strongly t-Baer notion. We show that every direct summand

of a strongly t-Baer module is strongly t-Baer. Necessary and sufficient conditions are given to show that a direct sum of strongly t-Baer modules is strongly t-Baer.

\section{Preliminaries}

Throughout all rings (not necessarily commutative rings) have identities and all modules are unital right modules. For the sake of completeness, we state some definitions and notations used throughout this paper. Let $M$ be a module over a $\operatorname{ring} R$. For submodules $N$ and $K$ of $M, N \leq K$ denotes $N$ is a submodule of $K$ and $\operatorname{End}(M)$ denotes the ring of right $R$-module endomorphisms of $M$. We denote by $r_{M}($.$) the right annihilator of a subset of \operatorname{End}(M)$ with elements from $M$. In what follows, by $\leq^{\oplus}$ and $\leq^{e s s}$ we denote, respectively, a module direct summand and an essential submodule of $M$. The symbols $\mathbb{Z}, \mathbb{Z}_{n}$ and $\mathbb{Q}$ stand for the ring of integers, the ring of residues modulo $n$ and the ring of rational numbers, respectively. 
Recall that the singular submodule $Z(M)$ of a module $M$ is the set of $m \in M$ with $\mathrm{r}_{R}(m) \leq{ }^{\text {ess }} R_{R}$, or equivalently, $m I=0$ for some essential right ideal $I$ of $R$. The second singular (or Goldie torsion) submodule $Z_{2}(M)$ is the submodule of $M$ which is defined by $Z\left(\frac{M}{Z(M)}\right)=\frac{Z_{2}(M)}{Z(M)}$. If $N$ is a submodule of $M$, then $Z(N)=Z(M) \cap N$ and so $Z_{2}(N)=Z_{2}(M) \cap N$. A module $M$ is called singular if $Z(M)=M$ and nonsingular if $Z(M)=0$. A module $M$ is called $Z_{2}$-torsion if $Z_{2}(M)=M$. If $M_{i}$ are $R$-modules $(i \in I)$, then $Z\left(\oplus_{i \in I} M_{i}\right)=\oplus_{i \in I} Z\left(M_{i}\right)$ and so $Z_{2}\left(\oplus_{i \in I} M_{i}\right)=\oplus_{i \in I} Z_{2}\left(M_{i}\right)$.

Definition 2.1. $\quad$ (a) An $R$-module $M$ is called strongly extending if each submodule of $M$ is essential in a fully invariant direct summand of $M([9])$.

(b) A module $M$ is said to be Abelian Baer, if for any left ideal $I$ of $\operatorname{End}(M)$, $\mathrm{r}_{M}(I)$ is a fully invariant direct summand of $M([14])$.

(c) A submodule $N$ of $M$ is called t-essential in $M$ if for every submodule $N^{\prime}$ of $M, N \cap N^{\prime} \leq Z_{2}(M)$ implies that $N^{\prime} \leq Z_{2}(M)$. The notation $N \leq^{\text {tess }} M$ denotes that $N$ is t-essential in $M$ ([3]).

(d) A submodule $C$ is called $t$-closed (resp. closed) if $C$ has no t-essential (resp. essential) extension in $M$. The symbol $C \leq^{t c} M$ denotes that $C$ is a t-closed submodule of $M([3])$.

(e) A module $M$ is called t-extending if every t-closed submodule of $M$ is a direct summand of $M([3])$.

(f) An $R$-module $M$ is said to be $t$-Baer, if $\mathrm{t}_{M}(I)=\left\{m \in M \mid I m \subseteq Z_{2}(M)\right\}$ is a direct summand of $M$ for each left ideal $I$ of $\operatorname{End}(M)([3])$.

(g) An $R$-module $M$ is said to be strongly t-Rickart, if $\mathrm{t}_{M}(\phi)$ is a fully invariant direct summand of $M$ for each $\phi \in \operatorname{End}(M)$. An $R$-module $M$ is said to be strongly Rickart, if $\operatorname{Ker}(\phi)$ is a fully invariant direct summand of $M$ for each $\phi \in \operatorname{End}(M)([8,9])$.

(h) An $R$-module $M$ is said to have summand intersection property (SIP), if the intersection of any two direct summands is a direct summand of $M$. $M$ is said to have the strongly summand intersection property (SSIP) if the intersection of any family of direct summands is a direct summand of $M$ ([1]).

(i) The module $M$ is called weakly co-Hopfian $(\mathrm{wcH})$ if every injective endomorphism has an essential image ([10]).

(l) An $R$-module $M$ is called densely co-Hopfian (dcH) if for all injective endomorphisms $f$ of $M, f(M)$ is a t-essential submodule of $M([2])$. 
(k) An idempotent $e \in R$ is called left semicentral if $r e=e r e$, for each $r \in R$, equivalently, $e R$ is an ideal of $\mathrm{R}$. The set of all semicentral idempotents of $R$ will be denoted by $S_{l}(R)$. If $e^{2}=e \in \operatorname{End}(M)$, then $e \in S_{l}(\operatorname{End}(M))$ if and only if $e M$ is a fully invariant direct summand $([6,7])$.

It is known from [2, Proposition 1.1] that

$$
Z_{2}(M)=\left\{m \in M \mid m I=0 \text { for some } I \leq^{\text {tess }} R_{R}\right\} .
$$

We need the following propositions proved in [9, Theorem 2.4], [3, Proposition 2.2], [3, Proposition 2.6], [3, Corollary 2.8], [3, Proposition 2.9] and [4, Lemma 2.3], respectively.

Proposition 2.2. Let $M$ be a module.

(a) $M$ is strongly extending if and only if it is extending and each direct summand of $M$ is fully invariant.

(b) The following statements are equivalent for a submodule $N$ of $M$.

(i) $N \leq^{\text {tess }} M$;

(ii) $N+Z_{2}(M) \leq \leq^{e s s} M$;

(iii) $\frac{N+Z_{2}(M)}{Z_{2}(M)} \leq$ ess $\frac{M}{Z_{2}(M)}$;

(iv) $\frac{M}{N}$ is $Z_{2}$-torsion.

(c) Let $C$ be a submodule of $M$. The following statements are equivalent:

(i) $C$ is t-closed in $M$;

(ii) $C$ contains $Z_{2}(M)$ and $C$ is closed in $M$;

(iii) $\frac{M}{C}$ is nonsingular.

(d) Let $L \subseteq K$ be two submodules of $M$. If $L$ is $t$-closed in $K$ and $K$ is $t$-closed in $M$, then $L$ is $t$-closed in $M$.

(e) Let $C \leq M$. If $C^{\prime} \leq{ }^{t c} M$, then $C \cap C^{\prime} \leq{ }^{t c} C$.

Lemma 2.3. Let $M$ be a module. Then every submodule $N$ of $M$ is contained in a t-closed submodule $C$ of $M$, where $N \leq{ }^{\text {tess }} C$.

\section{Strongly t-extending modules}

We start this section with the following definition.

Definition 3.1. An $R$-module $M$ is called strongly t-extending if for each submodule $N$ of $M$, there exists a fully invariant direct summand of $M$ containing $N$ as a t-essential submodule.

Clearly, every $Z_{2}$-torsion module is strongly t-extending. Moreover, every strongly extending module is strongly t-extending. 
The next result gives several equivalent conditions for a module to be strongly t-extending.

Theorem 3.2. The following are equivalent for an $R$-module $M$.

(1) $M$ is strongly t-extending;

(2) Every $t$-closed submodule of $M$ is a fully invariant direct summand;

(3) $M$ is t-extending and each direct summand of $M$ which contains $Z_{2}(M)$ is fully invariant;

(4) $M=Z_{2}(M) \oplus M^{\prime}$, where $M^{\prime}$ is a strongly extending module;

(5) Every submodule of $M$ which contains $Z_{2}(M)$ is essential in a fully invariant direct summand;

(6) Every submodule of $M$ which contains $Z_{2}(M)$ is t-essential in a fully invariant direct summand;

(7) For every submodule $A$ of $M, N$ is a fully invariant direct summand of $M$, where $\frac{N}{A}=Z_{2}\left(\frac{M}{A}\right)$;

(8) For every submodule $A$ of $M$, there exists a decomposition $\frac{M}{A}=\frac{N}{A} \oplus \frac{N^{\prime}}{A}$ such that $N$ is a fully invariant direct summand of $M$ and $N^{\prime} \leq{ }^{\text {tess }} M$.

Proof. (1) $\Rightarrow(2)$ Let $N$ be a t-closed submodule of $M$. Since $M$ is strongly textending, $N \leq{ }^{\text {tess }} F$, where $F$ is a fully invariant direct summand of $M$. So $N=F$ and hence $N$ is fully invariant direct summand of $M$.

$(2) \Rightarrow(3)$ Since every t-closed submodule of $M$ is a direct summand, $M$ is textending. We will prove that each direct summand of $M$ which contains $Z_{2}(M)$ is fully invariant. Let $K \leq^{\oplus} M$ which contains $Z_{2}(M)$. Since $K \leq^{\oplus} M, K$ is closed in $M$. By Proposition 2.2(c), $K$ is t-closed in $M$ and so by (2), $K$ is fully invariant.

(3) $\Rightarrow$ (4) Since $M$ is t-extending, $M=Z_{2}(M) \oplus M^{\prime}$, where $M^{\prime}$ is extending (by [3, Theorem 2.11]). We will prove that $M^{\prime}$ is strongly extending. By Proposition 2.2 (a), it suffices to show that each direct summand of $M^{\prime}$ is fully invariant in $M^{\prime}$. Let $K \leq^{\oplus} M^{\prime}$. Hence $K \oplus Z_{2}(M) \leq^{\oplus} M$ and contains $Z_{2}(M)$. By (3), $K \oplus Z_{2}(M)$ is fully invariant in $M$. We will prove that $K$ is fully invariant in $M^{\prime}$. Let $f \in$ $\operatorname{End}\left(M^{\prime}\right)$, so $1_{Z_{2}(M)} \oplus f \in \operatorname{End}(M)$. Thus $\left(1_{Z_{2}(M)} \oplus f\right)\left(Z_{2}(M) \oplus K\right) \subseteq Z_{2}(M) \oplus K$ because $Z_{2}(M) \oplus K$ is fully invariant in $M$. Therefore $Z_{2}(M) \oplus f(K) \subseteq Z_{2}(M) \oplus K$. Hence $f(K) \subseteq K$ and $K$ is fully invariant in $M^{\prime}$.

(4) $\Rightarrow(5)$ Let $K$ be a submodule of $M$ which contains $Z_{2}(M)$. By modular law, $K=Z_{2}(M) \oplus\left(K \cap M^{\prime}\right)$. Since $M^{\prime}$ is strongly extending, $K \cap M^{\prime} \leq^{\text {ess }}$ $N$ for some fully invariant direct summand $N$ of $M^{\prime}$. As $N \leq M^{\prime}, Z_{2}(M) \oplus$ $N \leq{ }^{\oplus} M$. Since $K \cap M^{\prime} \leq^{\text {ess }} N, Z_{2}(M) \oplus\left(K \cap M^{\prime}\right) \leq{ }^{\text {ess }} Z_{2}(M) \oplus N$. We will prove that $Z_{2}(M) \oplus N$ is a fully invariant direct summand of $M$. By (4), 
$M=Z_{2}(M) \oplus M^{\prime}$. Hence $\operatorname{End}(M)=\left(\begin{array}{cc}\operatorname{End}\left(Z_{2}(M)\right) & \operatorname{Hom}\left(M^{\prime}, Z_{2}(M)\right) \\ \operatorname{Hom}\left(Z_{2}(M), M^{\prime}\right) & \operatorname{End}\left(M^{\prime}\right)\end{array}\right)=$ $\left(\begin{array}{cc}\operatorname{End}\left(Z_{2}(M)\right) & \operatorname{Hom}\left(M^{\prime}, Z_{2}(M)\right) \\ 0 & \operatorname{End}\left(M^{\prime}\right)\end{array}\right) \quad$ (because $\left.\operatorname{Hom}\left(Z_{2}(M), M^{\prime}\right)=0\right)$. Therefore for each $f \in \operatorname{End}(M)$, we have $f\left(Z_{2}(M) \oplus N\right) \subseteq Z_{2}(M) \oplus N$, as $N$ is fully invariant in $M^{\prime}$. Hence $K$ is essential in a fully invariant direct summand of $M$.

$(5) \Rightarrow(6)$ It is clear from Proposition 2.2(b).

$(6) \Rightarrow(7)$ Let $A$ be a submodule of $M$ and consider a submodule $N$ defined by $N / A=Z_{2}(M / A)$. Since $\frac{M}{N} \cong \frac{\frac{M}{A}}{Z_{2}\left(\frac{M}{A}\right)}$ is nonsingular, $N$ is a t-closed submodule of $M$, by Proposition 2.2(c). By (6), $N$ is t-essential in a fully invariant direct summand of $M$ and so $N$ is a fully invariant direct summand of $M$.

$(7) \Rightarrow(8)$ Let $A$ be a submodule of $M$. By (7), there exists a fully invariant direct summand $N$ of $M$, say $M=N \oplus K$, where $\frac{N}{A}=Z_{2}\left(\frac{M}{A}\right)$. Thus $\frac{M}{A}=\frac{N}{A} \oplus \frac{K+A}{A}$. Since $\frac{M}{K+A} \cong \frac{\frac{M}{A}}{\frac{K+A}{A}} \cong \frac{N}{A}=Z_{2}\left(\frac{M}{A}\right)$, we have $\frac{M}{K+A}$ is $Z_{2}$-torsion, hence $K+A \leq$ tess $M$, by Proposition $2.2(\mathrm{~b})$.

$(8) \Rightarrow(1)$ Let $N$ be a submodule of $M$, then by Lemma $2.3, N \leq^{\text {tess }} C$ for some t-closed submodule $C$ of $M$. By (8), $\frac{M}{C}=\frac{K}{C} \oplus \frac{K^{\prime}}{C}$, where $K$ is a fully invariant direct summand of $M$ and $K^{\prime} \leq{ }^{\text {tess }} M$. Hence by Proposition $2.2(\mathrm{~b}), \frac{M}{K^{\prime}}$ is $Z_{2^{-}}$ torsion and so $\frac{K}{C} \cong \frac{\frac{M}{C}}{\frac{K^{\prime}}{C}} \cong \frac{M}{K^{\prime}}$ is $Z_{2}$-torsion. By Proposition $2.2(\mathrm{~b}) C \leq \leq^{\text {tess }} K$. Since $C$ is t-closed, $C=K$. Hence $N$ is t-essential in a fully invariant direct summand of $M$.

The next example shows that strongly t-extending modules need not be strongly extending.

Example 3.3. Consider $M=\mathbb{Z}_{n} \oplus \mathbb{Z}$ as a $\mathbb{Z}$-module where $n$ is a positive integer. By Theorem 3.2(4), $M$ is strongly t-extending. However $M$ is not strongly extending, by Proposition 2.2(a), because $\mathbb{Z}$ is not a fully invariant direct summand of $M$.

By Theorem 3.2, each strongly t-extending module is t-extending, but the converse does not hold in general, as the following example shows.

Example 3.4. Let $F$ be a field, $R=\left(\begin{array}{cc}F & F \\ 0 & F\end{array}\right)$ and $M$ be an arbitrary $R$-module. Then $Z_{2}(M) \oplus R$ is a t-extending module which is not strongly t-extending since $R_{R}$ is not strongly extending.

Theorem 3.5. If $M$ is a strongly t-extending module, then each direct summand of $M$ is strongly t-extending. 
Proof. Let $N \leq{ }^{\oplus} M$, say $M=N \oplus K$. Since $M$ is strongly t-extending, $M$ is t-extending, so are $N$ and $K$ by [3, Proposition 2.14]. We will show that each direct summand of $N$ contains $Z_{2}(N)$ is fully invariant. Let $H \leq^{\oplus} N$ and $Z_{2}(N) \subseteq H$. Since $K$ is t-extending, $K=Z_{2}(K) \oplus K^{\prime}$, where $K^{\prime}$ is extending (by [3, Theorem 2.11]). Since $H \leq^{\oplus} N, H \oplus Z_{2}(K)$ is a direct summand of $M$ which contains $Z_{2}(M)$, hence by Theorem 3.2(3), $H \oplus Z_{2}(K)$ is a fully invariant direct summand of $M$. We will prove that $H$ is fully invariant in $N$. If $f \in \operatorname{End}(N)$, then $1_{K} \oplus f \in \operatorname{End}(M)$. Thus $(1 \oplus f)\left(Z_{2}(K) \oplus H\right)=Z_{2}(K) \oplus f(H) \subseteq Z_{2}(K) \oplus H$. This implies that $f(H) \subseteq H$. Thus $N$ is t-extending and each direct summand of $N$ that contains $Z_{2}(N)$ is fully invariant, therefore $N$ is strongly t-extending, by Theorem 3.2(3).

In [9], it is shown that strongly extending modules are wcH. The following proposition shows that strongly t-extending modules are $\mathrm{dcH}$.

Theorem 3.6. If $M$ is strongly $t$-extending, then $M$ is $d c H$.

Proof. By Theorem 3.2, $M=Z_{2}(M) \oplus M^{\prime}$, where $M^{\prime}$ is strongly extending. Therefore $\frac{M}{Z_{2}(M)} \cong M^{\prime}$ is wcH. Let $f: M \rightarrow M$ be a monomorphism. Since $f\left(Z_{2}(M)\right) \subseteq Z_{2}(M), \bar{f}: \frac{M}{Z_{2}(M)} \rightarrow \frac{M}{Z_{2}(M)}$ with $\bar{f}\left(m+Z_{2}(M)\right)=f(m)+Z_{2}(M)$ is a homomorphism. Let $\bar{f}\left(m+Z_{2}(M)\right)=f(m)+Z_{2}(M)=0$. So $f(m) \in Z_{2}(M)$ and $f(m) I=0$ for some $I \leq^{\text {tess }} R_{R}$. Since $f$ is a monomorphism, $m I=0$, and hence $m \in Z_{2}(M)$. Thus $\bar{f}$ is a monomorphism. Since $\frac{M}{Z_{2}(M)}$ is wcH, $\bar{f}\left(\frac{M}{Z_{2}(M)}\right) \leq{ }^{\text {ess }}$ $\frac{M}{Z_{2}(M)}$. So $\frac{f(M)+Z_{2}(M)}{Z_{2}(M)} \leq^{e s s} \frac{M}{Z_{2}(M)}$. By Proposition $2.2(\mathrm{~b}), f(M) \leq^{\text {tess }} M$, hence $M$ is dcH.

Proposition 3.7. If $M$ is a strongly t-extending module, then $M$ has SSIP for direct summands that contain $Z_{2}(M)$.

Proof. Suppose that $M$ is strongly t-extending and let $\left\{M_{i}\right\}_{i \in I}$ be a family of direct summands of $M$ that contain $Z_{2}(M)$ and $M_{i}=e_{i} M$ for some idempotent $e_{i}$ of $\operatorname{End}(M)$, for all $i \in I$. If $\cap_{i \in I} M_{i}=0$, then there is nothing to prove. Suppose that $\cap_{i} M_{i} \neq 0$ and so $\cap_{i} M_{i} \leq^{\text {tess }} e M$ for some $e \in S_{l}(\operatorname{End}(M))$. Since $Z_{2}(M) \subseteq \cap_{i} M_{i}$, we have $\cap_{i} M_{i} \leq^{\text {ess }} e M$ by Proposition 2.2(b). Therefore for each $i \in I,\left(1-e_{i}\right) M \cap e M=0$, whence $e M \subseteq e_{i} M$. Thus $\cap_{i} M_{i}=e M$ and $M$ has SSIP for $\left\{M_{i}\right\}_{i \in I}$.

In the following, for a free module $F, \operatorname{rank}(F)$ denotes the minimum cardinality of any basis of $F$.

Theorem 3.8. Let $R$ be a ring and $F$ be a free $R$-module. The following are equivalent. 
(1) $F$ is strongly t-extending;

(2) (i) $R_{R}$ is strongly t-extending and $\operatorname{rank}(F)=1$, or

(ii) $R$ is $Z_{2}$-torsion.

Proof. $(1) \Rightarrow(2)$ Let $F$ be a free $R$-module and strongly t-extending with $\operatorname{rank}(F) \geq$ 2. By Theorem 3.5, $R$ is strongly t-extending. Hence by Theorem 3.2, $R=$ $Z_{2}(R) \oplus R^{\prime}$. Let $F=R^{(I)}$, then $F=Z_{2}(R)^{(I)} \oplus R^{\prime(I)}$ where $R^{\prime(I)}$ is strongly extending. Hence its direct summands must be fully invariant. Therefore $\operatorname{Hom}\left(R^{\prime}, R^{\prime}\right)=0$ and so $R^{\prime}=0$. Thus $R$ is $Z_{2}$-torsion.

$(2) \Rightarrow(1)$ It is clear.

Lemma 3.9. Let $M$ be a module. If $K \leq N$ is a fully invariant submodule of $M$ and $N \leq{ }^{\oplus} M$, then $K$ is fully invariant in $N$.

Proof. Let $K \leq N$ and $N \leq{ }^{\oplus} M$. Hence there exists $e^{2}=e \in \operatorname{End}(M)$ such that $N=e M$ and so $\operatorname{End}(N)=e \operatorname{End}(M) e$. Let $f \in \operatorname{End}(N)=e \operatorname{End}(M) e$. So there exists $g \in \operatorname{End}(M)$ such that $f=e g e$. Hence $f(K)=e g e(K)=e g(K) \subseteq e K=K$ because $K$ is fully invariant in $M$. Thus $K$ is fully invariant in $N$.

In general, a direct sum of strongly t-extending modules need not be a strongly t-extending module, as the following example shows.

Example 3.10. Suppose that $M_{1}=\mathbb{Z}_{p} \oplus \mathbb{Z}$ and $M_{2}=\mathbb{Z}_{q} \oplus \mathbb{Q}$, where $p, q$ are integer numbers. Consider $M_{1}$ and $M_{2}$ as $\mathbb{Z}$-modules. By Theorem 3.2, $M_{1}$ and $M_{2}$ are strongly t-extending. Since $\mathbb{Z}$ is not fully invariant in $\mathbb{Q} \oplus \mathbb{Z}$, the $\mathbb{Z}$-module $\mathbb{Q} \oplus \mathbb{Z}$ is not strongly extending by Proposition 2.2(a). Thus $M_{1} \oplus M_{2}$ is not strongly t-extending as a $\mathbb{Z}$-module by Theorem 3.2.

We can, however, provide some necessary conditions for a direct sum of any two modules to be strongly t-extending.

Proposition 3.11. Let $M=M_{1} \oplus M_{2}$. Then $M$ is strongly t-extending if and only if each $t$-closed submodule $K$ of $M$ with $K \cap M_{1} \subseteq Z_{2}(M)$ or $K \cap M_{2} \subseteq Z_{2}(M)$ is a fully invariant direct summand of $M$.

Proof. The necessity is clear. For the sufficiency, let $K$ be a t-closed submodule in $M$. By Lemma 2.3, there exists a t-closed submodule $L$ of $K$ such that $K \cap$ $M_{1} \leq{ }^{\text {tess }} L$. Since $L \leq{ }^{t c} K \leq \leq^{t c} M, L$ is t-closed in $M$ by Proposition 2.2(d). As $\left(K \cap M_{1}\right) \cap\left(M_{2} \cap L\right)=0$ and $K \cap M_{1} \leq^{\text {tess }} L$, we have $M_{2} \cap L \subseteq Z_{2}(L) \subseteq Z_{2}(M)$. By assumption $L$ is a fully invariant direct summand of $M$, say $M=L \oplus L^{\prime}$. By modular law $K=L \oplus\left(K \cap L^{\prime}\right)$. By Lemma 2.3, there exists a t-closed submodule 
$N$ of $K$ such that $K \cap L^{\prime} \leq{ }^{\text {tess }} N$. Since $N \leq{ }^{t c} K \leq{ }^{t c} M, N$ is t-closed in $M$. As $K \cap M_{1} \subseteq L,\left(K \cap M_{1}\right) \cap L^{\prime}=0$. So $\left(N \cap M_{1}\right) \cap\left(L^{\prime} \cap K\right)=0$, thus $N \cap M_{1} \subseteq Z_{2}(N) \subseteq Z_{2}(M)$. Therefore $N$ is a fully invariant direct summand of $M$, say $M=N \oplus N^{\prime}$. Since $K=L \oplus\left(K \cap L^{\prime}\right)$, using modular law implies $N=(N \cap L) \oplus\left(K \cap L^{\prime}\right)$. Thus $M=(N \cap L) \oplus\left(K \cap L^{\prime}\right) \oplus N^{\prime}$. By modular law $L^{\prime}=\left(K \cap L^{\prime}\right) \oplus T$ for some $T \leq M$. Hence $M=L \oplus L^{\prime}=L \oplus\left(K \cap L^{\prime}\right) \oplus T=K \oplus T$. It remains to show that $K$ is fully invariant. First we show that $N$ and $L$ are strongly t-extending. Let $U \leq{ }^{t c} N$. As $U \leq{ }^{t c} N \leq{ }^{t c} M, U \leq{ }^{t c} M$. Since $N \cap M_{1} \subseteq Z_{2}(M)$, we have $U \cap M_{1} \subseteq Z_{2}(M)$. By assumption $U$ is a fully invariant direct summand of $M$ and so is a direct summand of $N$. Since $N$ is a direct summand of $M$ and $U$ is a fully invariant direct summand of $M, U$ is fully invariant in $N$ by Lemma 3.9. Hence $N$ is strongly t-extending. Similarly $L$ is strongly t-extending. Since $N$ and $L$ are t-closed submodules of $M, Z_{2}(M) \subseteq L$ and $Z_{2}(M) \subseteq N$, by Proposition 2.2(c). So $Z_{2}(M) \subseteq L \cap N$. As $L \cap N \leq{ }^{\oplus} N$ and $N$ is strongly t-extending, $L \cap N$ is strongly t-extending by Theorem 3.5, and so we have $L \cap N=Z_{2}(M) \oplus Q$ by Theorem 3.2. Therefore $N=Z_{2}(M) \oplus Q \oplus\left(K \cap L^{\prime}\right)$. Since $N$ is strongly t-extending and $Z_{2}(M) \oplus\left(K \cap L^{\prime}\right) \leq{ }^{\oplus} N$ and $Z_{2}(M)=Z_{2}(N) \subseteq Z_{2}(M) \oplus\left(K \cap L^{\prime}\right)$, by Theorem $3.2, Z_{2}(M) \oplus\left(K \cap L^{\prime}\right)$ is fully invariant in $N$. As $N$ is a fully invariant direct summand of $M$ and $Z_{2}(M) \oplus\left(K \cap L^{\prime}\right)$ is fully invariant in $N, Z_{2}(M) \oplus\left(K \cap L^{\prime}\right)$ is fully invariant in $M$. As $L$ is strongly t-extending and $Z_{2}(L)=Z_{2}(M), L=$ $Z_{2}(M) \oplus L_{1}$ by Theorem 3.2. Now, we will show that $K$ is fully invariant in $M$. We have $K=L \oplus\left(K \cap L^{\prime}\right)=Z_{2}(M) \oplus L_{1} \oplus\left(K \cap L^{\prime}\right)$. Let $f \in \operatorname{End}(M)$, then $f(K)=f\left(Z_{2}(M) \oplus L_{1} \oplus\left(K \cap L^{\prime}\right)\right)=f\left(L_{1}\right)+f\left(Z_{2}(M) \oplus\left(K \cap L^{\prime}\right)\right) \subseteq f(L)+$ $f\left(Z_{2}(M) \oplus\left(K \cap L^{\prime}\right)\right) \subseteq L+\left(Z_{2}(M) \oplus\left(K \cap L^{\prime}\right)\right)=L \oplus\left(K \cap L^{\prime}\right)=K$. Hence $K$ is a fully invariant direct summand of $M$. Thus $M$ is strongly t-extending.

The next theorem gives a condition that a direct sum of two strongly t-extending modules is strongly t-extending.

Theorem 3.12. Let $M_{1}$ and $M_{2}$ be strongly t-extending modules and $M_{i}^{\prime} \leq M_{i}$ such that $M_{i}=Z_{2}\left(M_{i}\right) \oplus M_{i}^{\prime}$ for $i=1,2$. If $\operatorname{Hom}\left(N_{1}, M_{2}^{\prime}\right)=0$ and $\operatorname{Hom}\left(N_{2}, M_{1}^{\prime}\right)=0$ for each $N_{1} \leq M_{1}^{\prime}$ and $N_{2} \leq M_{2}^{\prime}$, then $M=M_{1} \oplus M_{2}$ is strongly t-extending.

Proof. Let $K$ be a t-closed submodule of $M$ such that $K \cap M_{1} \subseteq Z_{2}(M)$. By Proposition 2.2(c), $Z_{2}(M) \subseteq K$. Therefore $Z_{2}\left(M_{1}\right)=Z_{2}(M) \cap M_{1} \subseteq K \cap M_{1} \subseteq$ $Z_{2}(M) \cap M_{1}=Z_{2}\left(M_{1}\right)$ and so $K \cap M_{1}=Z_{2}\left(M_{1}\right)$. Thus $K \cap M_{1}^{\prime}=\left(K \cap M_{1}\right) \cap$ $M_{1}^{\prime}=Z_{2}\left(M_{1}\right) \cap M_{1}^{\prime}=0$. By modular law, $K=Z_{2}(M) \oplus\left(K \cap\left(M_{1}^{\prime} \oplus M_{2}^{\prime}\right)\right)$. Put $C=K \cap\left(M_{1}^{\prime} \oplus M_{2}^{\prime}\right)$. Let $\pi_{i}: M_{1}^{\prime} \oplus M_{2}^{\prime} \rightarrow M_{i}^{\prime}$ be the canonical projection for $i=1,2$. As $\operatorname{Ker}\left(\left.\pi_{2}\right|_{C}\right)=\operatorname{Ker}\left(\pi_{2}\right) \cap C=M_{1}^{\prime} \cap C=M_{1}^{\prime} \cap K=$ 
0 , we have a monomorphism $\left.\pi_{2}\right|_{C}: C \rightarrow M_{2}^{\prime}$. Since $\left.\pi_{1}\right|_{C}=\pi_{1}\left(\left(\left.\pi_{2}\right|_{C}\right)^{-1}\right):$ $\pi_{2}(C) \rightarrow M_{1}^{\prime} \in \operatorname{Hom}\left(\pi_{2}(C), M_{1}^{\prime}\right)$ and by assumption $\operatorname{Hom}\left(\pi_{2}(C), M_{1}^{\prime}\right)=0$, we have $\pi_{1}(C)=0$. Thus $C \subseteq \operatorname{Ker}\left(\pi_{1}\right)=M_{2}^{\prime}$. By Proposition 2.2(e), $C$ is t-closed in $M_{1}^{\prime} \oplus M_{2}^{\prime}$ so $C$ is t-closed in $M_{2}^{\prime}$. Since $M_{2}^{\prime}$ is nonsingular and strongly extending, $C$ is a fully invariant direct summand of $M_{2}^{\prime}$. As $\operatorname{Hom}\left(M_{1}^{\prime}, M_{2}^{\prime}\right)=0$ and $\operatorname{Hom}\left(M_{2}^{\prime}, M_{1}^{\prime}\right)=0, \quad \operatorname{End}\left(M_{1}^{\prime} \oplus M_{2}^{\prime}\right)=\left(\begin{array}{cc}\operatorname{End}\left(M_{1}^{\prime}\right) & 0 \\ 0 & \operatorname{End}\left(M_{2}^{\prime}\right)\end{array}\right)$, therefore $M_{2}^{\prime}$ is fully invariant in $M_{1}^{\prime} \oplus M_{2}^{\prime}$. Thus $C$ is fully invariant in $M_{1}^{\prime} \oplus M_{2}^{\prime}$ by [5, Lemma 1.1]. Since $C \leq^{\oplus} M_{2}^{\prime} \leq^{\oplus} M_{1}^{\prime} \oplus M_{2}^{\prime}, C$ is a direct summand of $M_{1}^{\prime} \oplus M_{2}^{\prime} . \quad$ As $\quad \operatorname{End}(M)=\left(\begin{array}{cc}\operatorname{End}\left(Z_{2}(M)\right) & \operatorname{Hom}\left(M_{1}^{\prime} \oplus M_{2}^{\prime}, Z_{2}(M)\right) \\ \operatorname{Hom}\left(Z_{2}(M), M_{1}^{\prime} \oplus M_{2}^{\prime}\right) & \operatorname{End}\left(M_{1}^{\prime} \oplus M_{2}^{\prime}\right)\end{array}\right)=$ $\left(\begin{array}{cc}\operatorname{End}\left(Z_{2}(M)\right) & \operatorname{Hom}\left(M_{1}^{\prime} \oplus M_{2}^{\prime}, Z_{2}(M)\right) \\ 0 & \operatorname{End}\left(M_{1}^{\prime} \oplus M_{2}^{\prime}\right)\end{array}\right), K=Z_{2}(M) \oplus C$ is a fully invariant direct summand of $M$. Similarly, if $K \cap M_{2} \subseteq Z_{2}(M)$, then $K$ is a fully invariant direct summand of $M$. Therefore by Proposition 3.11, $M$ is strongly t-extending.

\section{Strongly t-Baer modules}

The purpose of this section is to introduce the concept of strongly t-Baer modules which are particular t-Baer modules, and study some basic properties of this new class of modules. For the rest of the article, $M$ is an $R$-module and $S=\operatorname{End}\left(M_{R}\right)$.

Definition 4.1. A module $M$ is called strongly t-Baer if $\mathrm{t}_{M}(I)$ is a fully invariant direct summand of $M$ for each left ideal $I$ of $S$.

Clearly every $Z_{2}$-torsion module is strongly t-Baer. Moreover, the notions of Abelian Baer and strongly t-Baer coincide for every nonsingular module. In particular, every Abelian Baer ring is strongly t-Baer (because every Abelian Baer ring is nonsingular).

The next theorem states some equivalent conditions for a strongly t-Baer module.

Theorem 4.2. The following statements are equivalent for a module $M$.

(1) $M$ is strongly $t$-Baer;

(2) $M$ is t-Baer and each direct summand which contains $Z_{2}(M)$ is fully invariant;

(3) $M=Z_{2}(M) \oplus M^{\prime}$, where $M^{\prime}$ is an Abelian Baer module;

(4) $M=Z_{2}(M) \oplus M^{\prime}$ and for each left ideal $I$ of $S, \mathrm{t}_{M}(I) \cap M^{\prime}$ is a fully invariant direct summand of $M^{\prime}$;

(5) For any left ideal $I$ of $S, \mathrm{t}_{M}(I)$ is t-essential in a fully invariant direct summand of $M$. 
Proof. $(1) \Rightarrow(2)$ Let $M$ be a strongly t-Baer module. Therefore $M$ is t-Baer. Let $K$ be a direct summand of $M$ which contains $Z_{2}(M)$. We will show that $K$ is fully invariant. Let $K=e M$ for some idempotent $e \in S$. Since $\mathrm{t}_{M}(1-e)=$ $\mathrm{t}_{M}(S(1-e))=e M=K$ and $M$ is strongly t-Baer, $K$ is fully invariant.

$(2) \Rightarrow(3)$ Let $M$ be a t-Baer module. Assume that each direct summand of $M$ which contains $Z_{2}(M)$ is fully invariant. Therefore $M=Z_{2}(M) \oplus M^{\prime}$. We show $M^{\prime}$ is Abelian Baer. Let $I^{\prime}$ be a left ideal of $S^{\prime}=\operatorname{End}\left(M^{\prime}\right)$. Set $A=\left\{1_{Z_{2}(M)} \oplus \phi \mid \phi \in I^{\prime}\right\}$ and $I=S A$. Then $\mathrm{t}_{M}(I)=Z_{2}(M) \oplus \mathrm{r}_{M^{\prime}}\left(I^{\prime}\right)$. As $M$ is t-Baer, $\mathrm{t}_{M}(I)$ is a direct summand of $M$. Therefore $\mathrm{r}_{M^{\prime}}\left(I^{\prime}\right) \leq{ }^{\oplus} M^{\prime}$. It suffices to show that $\mathrm{r}_{M^{\prime}}\left(I^{\prime}\right)$ is fully invariant. Since $Z_{2}(M) \subseteq \mathrm{t}_{M}(I), \mathrm{t}_{M}(I)$ is fully invariant by assumption. Let $f \in \operatorname{End}\left(M^{\prime}\right)$, then $1_{Z_{2}(M)} \oplus f \in S$. Hence $(1 \oplus f)\left(\mathrm{t}_{M}(I)\right)=Z_{2}(M) \oplus f\left(\mathrm{r}_{M^{\prime}}\left(I^{\prime}\right)\right) \subseteq$ $Z_{2}(M) \oplus \mathrm{r}_{M^{\prime}}\left(I^{\prime}\right)=\mathrm{t}_{M}(I)$. Thus $f\left(\mathrm{r}_{M^{\prime}}\left(I^{\prime}\right)\right) \subseteq \mathrm{r}_{M^{\prime}}\left(I^{\prime}\right)$. Therefore $\mathrm{r}_{M^{\prime}}\left(I^{\prime}\right)$ is a fully invariant direct summand of $M^{\prime}$ and $M^{\prime}$ is Abelian Baer.

(3) $\Rightarrow$ (4) Suppose that $M=Z_{2}(M) \oplus M^{\prime}$ where $M^{\prime}$ is Abelian Baer. Let $I$ be a left ideal of $S$ and $A^{\prime}=\left\{\left.\pi \phi\right|_{M^{\prime}} \mid \phi \in I\right\}$ (where $\pi$ is the canonical projection onto $\left.M^{\prime}\right)$ and $I^{\prime}=S^{\prime} A^{\prime}$ where $S^{\prime}=\operatorname{End}\left(M^{\prime}\right)$. Then $\mathrm{t}_{M}(I) \cap M^{\prime}=\mathrm{r}_{M^{\prime}}\left(I^{\prime}\right)$. Since $M^{\prime}$ is Abelian Baer, $\mathrm{t}_{M}(I) \cap M^{\prime}$ is a fully invariant direct summand of $M^{\prime}$.

$(4) \Rightarrow(1)$ Let $I$ be a left ideal of $S$. Since $Z_{2}(M) \subseteq \mathrm{t}_{M}(I)$, by modular law we have $\mathrm{t}_{M}(I)=Z_{2}(M) \oplus\left(\mathrm{t}_{M}(I) \cap M^{\prime}\right)$. By (4), $\mathrm{t}_{M}(I) \cap M^{\prime} \leq{ }^{\oplus} M^{\prime}$. Therefore $\mathrm{t}_{M}(I) \leq^{\oplus} M$. It remains to show that $\mathrm{t}_{M}(I)$ is fully invariant in $M$. As $M=Z_{2}(M) \oplus M^{\prime}$, we have $S=\left(\begin{array}{cc}\operatorname{End}\left(Z_{2}(M)\right) & \operatorname{Hom}\left(M^{\prime}, Z_{2}(M)\right) \\ \operatorname{Hom}\left(Z_{2}(M), M^{\prime}\right) & \operatorname{End}\left(M^{\prime}\right)\end{array}\right)=$ $\left(\begin{array}{cc}\operatorname{End}\left(Z_{2}(M)\right) & \operatorname{Hom}\left(M^{\prime}, Z_{2}(M)\right) \\ 0 & \operatorname{End}\left(M^{\prime}\right)\end{array}\right)$. Since $\mathrm{t}_{M}(I) \cap M^{\prime}$ is fully invariant in $M^{\prime}$ and $Z_{2}(M)$ is fully invariant in $M, \mathrm{t}_{M}(I)$ is fully invariant in $M$. Hence $M$ is strongly t-Baer.

$(1) \Rightarrow(5)$ It is clear.

$(5) \Rightarrow(1)$ Let $I$ be a left ideal of $S$. By $(5), \mathrm{t}_{M}(I) \leq{ }^{\text {tess }} K$ for some fully invariant direct summand of $M$. Since $Z_{2}(K) \subseteq Z_{2}(M) \subseteq \mathrm{t}_{M}(I), \mathrm{t}_{M}(I) \leq{ }^{\text {ess }} K$ by Proposition 2.2(b). Therefore for each $k \in K$, there exists $J \leq{ }^{\text {ess }} R_{R}$ such that $k J \subseteq \mathrm{t}_{M}(I)$. Hence for each $f \in I, f(k) J \subseteq Z_{2}(M)$. This implies that $f(k)+Z_{2}(M) \in Z\left(\frac{M}{Z_{2}(M)}\right)=0$. Thus $f(k) \in Z_{2}(M)$ for each $f \in I$. So $k \in \mathrm{t}_{M}(I)$ and $\mathrm{t}_{M}(I)=K$. Thus for each left ideal $I$ of $S, \mathrm{t}_{M}(I)$ is a fully invariant direct summand in $M$ and so $M$ is strongly t-Baer.

By Theorem 4.2(5), every strongly t-extending module is strongly t-Baer. The converse is not true in general. 
Example 4.3. (1) Let $R$ be a domain which is not right Ore. Since $R_{R}$ is not extending (see [5, Page 1410]), $R_{R}$ is not strongly extending. Let $N$ be an arbitrary right $R$-module. Then $M=R \oplus Z_{2}(N)$ is strongly $t$-Baer by Theorem 4.2, which is not a strongly t-extending module.

(2) Let $M=\mathbb{Z}_{n} \oplus \mathbb{Z}$, where $n$ is a positive integer. Then $M$ is a strongly $t$-Baer $\mathbb{Z}$-module which is not Abelian Baer.

Theorem 4.4. If $M$ is strongly $t$-Baer, then so is every direct summand of $M$.

Proof. Let $N$ be a direct summand of $M$, say $M=N \oplus K$. Since $M$ is strongly t-Baer, it is t-Baer, therefore each direct summand of $M$ is too, by [3, Theorem 3.6]. Thus $N$ is t-Baer. We will show that each direct summand of $N$ which contains $Z_{2}(N)$ is fully invariant in $N$. Let $W \leq^{\oplus} N$ and $Z_{2}(N) \subseteq W$. Since $K$ is t-Baer, $K=Z_{2}(K) \oplus K^{\prime}$. Thus $W \oplus Z_{2}(K) \leq^{\oplus} M$ and $Z_{2}(M) \subseteq W \oplus Z_{2}(K)$. By Theorem 4.2(2), $W \oplus Z_{2}(K)$ is fully invariant in $M$. Let $f \in \operatorname{End}(N)$, then $1_{K} \oplus f \in S$. Since $W \oplus Z_{2}(K)$ is fully invariant in $M,\left(1_{K} \oplus f\right)\left(W \oplus Z_{2}(K)\right) \subseteq W \oplus Z_{2}(K)$ and hence $f(W) \subseteq W$. Thus $W$ is fully invariant in $N$ and $N$ is strongly t-Baer.

Proposition 4.5. An R-module $M$ is strongly t-Baer if and only if $M$ is a strongly t-Rickart module and $M$ has the strongly summand intersection property (SSIP) for direct summands which contain $Z_{2}(M)$.

Proof. Let $M$ be a strongly t-Baer module. Then $M$ is strongly t-Rickart. Let $\left\{N_{j}\right\}_{j \in J}$ be a set of direct summands of $M$ which contain $Z_{2}(M)$. Assume that for each $j \in J, N_{j}=e_{j} M$ for some $e_{j}^{2}=e_{j} \in S$. Since $Z_{2}(M) \subseteq N_{j}, \mathrm{t}_{M}\left(1-e_{j}\right)=N_{j}$ for each $j \in J$. As $\cap_{j \in J} N_{j}=\cap_{j \in J} \mathrm{t}_{M}\left(1-e_{j}\right)=\mathrm{t}_{M}(I)$ where $I=\sum_{j \in J} S\left(1-e_{j}\right)$ and $M$ is strongly t-Baer, $\cap_{j \in J} N_{j} \leq^{\oplus} M$. Hence $M$ has SSIP for direct summands which contain $Z_{2}(M)$.

Conversely, let $I$ be a left ideal of $S$. Then $\mathrm{t}_{M}(I)=\cap_{\phi \in I} \mathrm{t}_{M}(\phi)$. Since $M$ is strongly t-Rickart, $\mathrm{t}_{M}(\phi)$ is a fully invariant direct summand of $M$. Since $M$ has SSIP for direct summands which contain $Z_{2}(M), \mathrm{t}_{M}(I) \leq{ }^{\oplus} M$. It remains to show that $\mathrm{t}_{M}(I)$ is fully invariant. Since for each $\phi \in I, \mathrm{t}_{M}(\phi)$ is fully invariant, $f\left(\mathrm{t}_{M}(I)\right)=f\left(\cap_{\phi \in I} \mathrm{t}_{M}(\phi)\right) \subseteq \cap_{\phi \in I} f\left(\mathrm{t}_{M}(\phi)\right) \subseteq \cap_{\phi \in I} \mathrm{t}_{M}(\phi)$ for each $f \in S$. Thus $\mathrm{t}_{M}(I)$ is a fully invariant direct summand of $M$. Therefore $M$ is strongly t-Baer.

Strongly $t$-Rickart modules are not strongly t-Baer as the following example shows.

Example 4.6. Let $S=\Pi_{i=1}^{\infty} \mathbb{Z}_{2}$ and $R=\left\{\left(a_{i}\right)_{i=1}^{\infty} \in S \mid a_{n}\right.$ is eventually constant $\}$. By [12, Example 4.1], $R_{R}$ is Rickart. Since $R$ is a commutative ring, it is strongly 
Rickart. Let $M$ be an arbitrary $R$-module, then $R \oplus Z_{2}(M)$ is a strongly t-Rickart $R$-module by [8, Theorem 4.2] which is not strongly $t$-Baer (because $R_{R}$ is not Baer).

Theorem 4.7. Let $M=\oplus_{i \in I} M_{i}$ be a direct sum of modules $M_{i}(i \in I)$ for some index set $I$. The following are equivalent.

(1) $M$ is strongly $t$-Baer.

(2) (i) For each $i \in I, M_{i}=Z_{2}\left(M_{i}\right) \oplus M_{i}^{\prime}$ where $M_{i}^{\prime}$ is Abelian Baer.

(ii) For each distinct $i, j \in I, \operatorname{Hom}\left(M_{i}^{\prime}, M_{j}^{\prime}\right)=0$.

Proof. (1) $\Rightarrow(2)$ (i) Let $M$ be a strongly t-Baer module. By Theorem 4.4, for each $i \in I, M_{i}$ is strongly t-Baer. Therefore $M_{i}=Z_{2}\left(M_{i}\right) \oplus M_{i}^{\prime}$ which $M_{i}^{\prime}$ is Abelian Baer by Theorem 4.2.

(ii) By (i), $M=\bigoplus_{i}\left(Z_{2}\left(M_{i}\right) \oplus M_{i}^{\prime}\right)=Z_{2}(M) \oplus\left(\oplus_{i} M_{i}^{\prime}\right)$. Since $M$ is strongly tBaer, $\oplus_{i} M_{i}^{\prime}$ is Abelian Baer. Thus each direct summand of $\oplus_{i} M_{i}^{\prime}$ is fully invariant. Therefore for each $i \in I, M_{i}^{\prime}$ is fully invariant in $\oplus_{i} M_{i}^{\prime}$. Hence for each distinct $i, j \in I, \operatorname{Hom}\left(M_{i}^{\prime}, M_{j}^{\prime}\right)=0$.

$(2) \Rightarrow(1)$ As $Z_{2}\left(M_{i}\right) \leq{ }^{\oplus} M_{i}$ for each $i \in I$ and $Z_{2}(M)=\oplus_{i} Z_{2}\left(M_{i}\right), Z_{2}(M) \leq^{\oplus}$ $M$, say $M=Z_{2}(M) \oplus M^{\prime}$ where $M^{\prime}=\oplus_{i} M_{i}^{\prime}$. Let $J$ be a left ideal of $S$. We show that $\mathrm{t}_{M}(J) \cap M^{\prime}$ is a fully invariant direct summand of $M^{\prime}$. Let $e$ be canonical projection from $M$ onto $M^{\prime}$. Then $\mathrm{t}_{M}(J) \cap M^{\prime}=\mathrm{r}_{M^{\prime}}\left(S^{\prime} J^{\prime}\right)=\mathrm{r}_{M^{\prime}}\left(J^{\prime}\right)$ where $J^{\prime}=\{$ efe $f \in J\}$ and $S^{\prime}=\operatorname{End}\left(\mathrm{M}^{\prime}\right)$. By (2)(ii), for each distinct $i, j \in I$, $\operatorname{Hom}\left(M_{i}^{\prime}, M_{j}^{\prime}\right)=0$, therefore for each $f \in J$, efe $=\oplus_{i} f_{i}^{\prime}$ where $f_{i}^{\prime} \in \operatorname{End}\left(M_{i}^{\prime}\right)$. Thus $r_{M^{\prime}}\left(J^{\prime}\right)=\oplus_{i} \mathrm{r}_{M_{i}^{\prime}}\left(J_{i}^{\prime}\right)$ where $J_{i}^{\prime}=\left\{f_{i}^{\prime} \in \operatorname{End}\left(M_{i}^{\prime}\right) \mid\right.$ efe $=\oplus_{i} f_{i}^{\prime}$ for each $\left.i \in I\right\}$. Since $M_{i}^{\prime}$ is Abelian Baer for each $i \in I, \mathrm{r}_{M_{i}^{\prime}}\left(J_{i}^{\prime}\right)$ is a fully invariant direct summand of $M_{i}^{\prime}$. Therefore $\mathrm{t}_{M}(J) \cap M^{\prime}=\mathrm{r}_{M^{\prime}}\left(S^{\prime} J^{\prime}\right)$ is a fully invariant direct summand of $M^{\prime}$. Hence Theorem 4.2(4) completes the proof.

Acknowledgment. The authors are deeply indebted to the referee for many helpful comments and suggestions for the improvement of this article.

\section{References}

[1] M. Alkan and A. Harmanci, On summand sum and summand intersection property of modules, Turkish J. Math., 26(2) (2002), 131-147.

[2] Sh. Asgari and A. Haghany, Densely co-Hopfian modules, J. Algebra Appl., 9(6) (2010), 989-1000.

[3] Sh. Asgari and A. Haghany, t-Extending modules and t-Baer modules, Comm. Algebra, 39 (2011), 1605-1623.

[4] Sh. Asgari, A. Haghany and A. R. Rezaei, Modules whose t-closed submodules have a summand as a complement, Comm. Algebra, 42 (2014), 5299-5318. 
[5] G. F. Birkenmeier, B. J. Müller and S. T. Rizvi, Modules in which every fully invariant submodule is essential in a direct summand, Comm. Algebra, 30(3) (2002), 1395-1415.

[6] G. F. Birkenmeier, J. K. Park and S. T. Rizvi, Generalized triangular matrix rings and the fully invariant extending property, Rocky Mountain J. Math., 32 (2002), $1299-1319$.

[7] G. F. Birkenmeier, J. K. Park and S. T. Rizvi, Modules with fully invariant submodules essential in fully invariant summands, Comm. Algebra, 30(4) (2002), 1833-1852.

[8] S. Ebrahimi Atani, M. Khoramdel and S. Dolati Pish Hesari, T-Rickart modules, Colloq. Math., 128(1) (2012), 87-100.

[9] S. Ebrahimi Atani, M. Khoramdel and S. Dolati Pish Hesari, On strongly extending modules, Kyungpook Math. J., 54 (2014), 237-247.

[10] A. Haghany and M. R. Vedadi, Modules whose injective endomorphisms are essential, J. Algebra, 243 (2001), 765-779.

[11] I. Kaplansky, Rings of Operators, W. A. Benjamin, Inc., New YorkAmsterdam, 1968.

[12] G. Lee, S. T. Rizvi and C. S. Roman, Rickart modules, Comm. Algebra, 38 (2010), 4005-4027.

[13] S. T. Rizvi and C. S. Roman, Baer and quasi-Baer modules, Comm. Algebra, 32(1) (2004), 103-123.

[14] S. T. Rizvi and C. S. Roman, On $\mathcal{K}$-nonsingular modules and applications, Comm. Algebra, 35 (2007), 2960-2982.

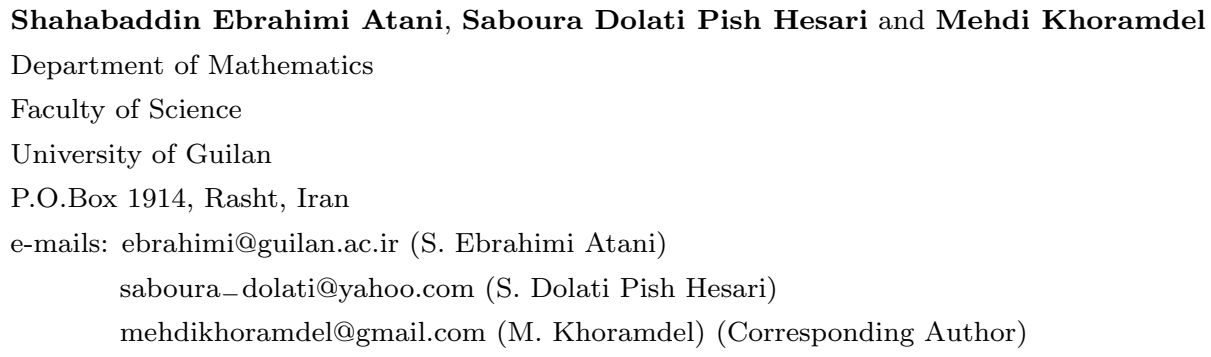

